\title{
Incidence and risk factors for blood transfusion in simultaneous bilateral total hip arthroplasty
}

\author{
Xing Wang, MD®, Qiang Huang, MD(D), Fuxing Pei, MD(D) \\ Department of Orthopaedic Surgery, West China Hospital, Chengdu, Sichuan Province, China
}

A large proportion of hip joint diseases develop bilaterally, and studies have shown that about 15 to $17 \%$ of patients undergoing one total hip arthroplasty (THA) require a second arthroplasty in the contralateral side. ${ }^{[1]}$ Bilateral THA can be usually performed simultaneous bilateral hip arthroplasty (SimBTHA) or staged bilateral total hip arthroplasties (StBTHA). ${ }^{[2]}$

Previous studies have demonstrated that SimBTHA has the advantages of shorter anesthesia and operation time, lower cost, and shorter recovery time. ${ }^{[3,4]}$ Although it has become increasingly preferred by physicians and patients recently, ${ }^{[5,6]}$ it often has more blood loss than StBTHA, with a higher incidence of blood transfusion. ${ }^{[3,7,8]}$ Therefore, due to the need for large amounts of blood transfusions, this may increase the risk of complications such as surgical site infection, venous thromboembolism, prolonged hospital stay, and mortality. ${ }^{[9-13]}$ Surgeons and patients are often hesitant to perform bilateral

Received: July 04, 2021

Accepted: August 18, 2021

Published online: November 19, 2021

Correspondence: Qiang Huang, MD. Department of Orthopaedic Surgery, West China Hospital, Chengdu, Sichuan Province, 610041 China.

E-mail: huangqiang4325@126.com

Doi: $10.52312 / j d r s .2021 .333$

Citation: Wang $X$, Huang $Q$, Pei F. Incidence and risk factors for blood transfusion in simultaneous bilateral total hip arthroplasty. Jt Dis Relat Surg 2021;32(3):590-597.

(C2021 All right reserved by the Turkish Joint Diseases Foundation

This is an open access article under the terms of the Creative Commons Attribution-NonCommercial License, which permits use, distribution and reproduction in any medium, provided the original work is properly cited and is not used for commercial purposes (http://creativecommons.org/licenses/by-nc/4.0/).

\section{ABSTRACT}

Objectives: This study aims to investigate the incidence and risk factors for blood transfusion in patients undergoing simultaneous bilateral total hip arthroplasty (SimBTHA).

Patients and methods: Between January 2011 and June 2021, a total of 341 patients (289 males, 52 females; median age: 53 years; range, 43 to 66 years) who underwent SimBTHA were retrospectively analyzed. The patients were divided into two groups as those who did and did not require allogeneic blood transfusion. Univariate and multivariate logistic regression models were used to identify independent risk factors for transfusion.

Results: The incidence of transfusion in SimBTHA was $12.9 \%$. We found that a higher preoperative hemoglobin level (odds ratio [OR]: 0.91; 95\% confidence interval [CI]: 0.86-0.96), tranexamic acid (TXA) use (OR: 0.25 ; 95\% CI: $0.09-0.58$ ), and intraoperative autologous blood transfusion from a closed suction drainage system (OR: 0.30; 95\% CI: 0.09-0.80) were protective against the need for transfusion in patients after SimBTHA. In addition, drainage use (OR: 3.52; 95\% CI: 1.49-8.32) and intraoperative blood loss (OR: 1.17; 95\% CI: 1.04-1.33) were the independent risk factors for blood transfusion.

Conclusion: The risk factors for transfusion after SimBTHA should be evaluated to establish specific, personalized transfusion risk assessments for each individual patient. For SimBTHA, the main risk factors include intraoperative bleeding and the use of drainage tube, while higher preoperative hemoglobin levels, TXA use, and autologous blood transfusion from a closed suction drainage system may reduce transfusion risk.

Keywords: Blood transfusion, risk factors, simultaneous bilateral total hip arthroplasty.

surgery simultaneously. How to minimize the transfusion rate after SimBTHA is an important concern for surgeons. In the present study, we aimed to evaluate the incidence and risk factors for blood transfusion in patients undergoing SimBTHA.

\section{PATIENTS AND METHODS}

This single-center, retrospective study was conducted at West China Hospital, Department of Orthopaedic 
Surgery between January $1^{\text {st }}, 2011$ and June 31 ${ }^{\text {st }}, 2021$. A total of 341 patients ( 289 males, 52 females; median age: 53 years; range, 43 to 66 years) who underwent SimBTHA were included in the study. A patient was considered eligible for simBTHA, if both hips showed lesions and symptoms. In our routine practice, we discouraged simultaneous bilateral surgery, if any of the following criteria are met: age $>80$ years, American Society of Anesthesiologists (ASA) Class II-IV with medical comorbidities including active ischemic heart disease or decreased left ventricular function $<45 \%$, moderate-to-severe pulmonary disease, renal insufficiency with glomerular filtration rate (GFR) $<60 \mathrm{~mL} / \mathrm{min} / 1.73 \mathrm{~m}^{2}$, chronic liver disease, poorly controlled diabetes mellitus with hemoglobin A1c (HbA1c) $>7 \%$, cerebrovascular disease with history of cerebrovascular accident, and peripheral vascular disease with history of stents or bypass. In our clinic, all patients undergo optimization by an internist and anesthesiologist, who must agree on the patient's eligibility for SimBTHA. All patients also undergo a preoperative echocardiogram and consultation with a cardiologist, if they are in ASA Class II or greater or have any concerning symptoms preoperatively.

Medical data of all patients were retrieved from the hospital database, and only patients whose surgical name included "bilateral THA" were included. We excluded those with incomplete information, coagulation disorders, and undergoing autologous blood predonation before surgery. The study protocol was approved by the West China Hospital Ethics Committee (Date/No: 2021/268, Date: 07/01/2021). The study was conducted in accordance with the principles of the Declaration of Helsinki.

All surgeries were performed by a single surgical team following standard procedures through a posterolateral approach. No patient received preoperative erythropoietin, and preoperative autologous donation did not occur. In practice, as the concept of the surgical team evolved, there was a gradual shift over the decade from the routine use of drainage tubes and no tranexamic acid (TXA) to the routine use of TXA and no drainage tubes. After 2016, the TXA administration for SimBTHA became a uniformly adopted practice, while drainage tubes were no longer routinely used. The TXA $(20 \mathrm{mg} / \mathrm{kg})$ was intravenously injected $\sim 5$ to 10 min before skin incision, and $1 \mathrm{~g}$ of TXA was added at 3 and $6 \mathrm{~h}$, respectively after surgery. The use of intraoperative autologous blood transfusion from a closed suction drainage system was determined by the operating surgeon based on the expected intraoperative blood loss of the patient. The recommended postoperative thromboprophylaxis included chemical (starting $6 \mathrm{~h}$ after surgery, patients without contraindications were given 2000 AxaIU $(0.2 \mathrm{~mL})$ low-molecular-weight heparin of subcutaneously once a day. After discharge, patients were given oral rivaroxaban $5 \mathrm{mg}$ once a day for two weeks) and mechanical methods (upon returning to the ward from surgery, patients were treated with intermittent pneumatic compression device for $12 \mathrm{~h})$. All patients received unified rehabilitation after surgery.

Transfusion was defined as any allogeneic red blood cell or plasma given within $72 \mathrm{~h}$ after the commencement of surgery. Blood transfusion was performed, if the hemoglobin level was $<70 \mathrm{~g} / \mathrm{L}$ or 70 to $100 \mathrm{~g} / \mathrm{L}$ with symptoms of anemia (including altered mental status, palpitations or shortness of breath not attributable to other causes). All intra- and postoperative blood transfusion events were recorded. We collected patient demographic characteristics from medical records, including sex, age, body mass index, smoking history, alcohol consumption history, and major diagnoses. Comorbidities included hypertension and diabetes mellitus. Preoperative laboratory values included hemoglobin, hematocrit, platelets, activated partial thromboplastin time (APTT), prothrombin time (PT), albumin, interleukin-6 (IL-6), C-reactive protein (CRP), and erythrocyte sedimentation rate (ESR). Surgical variables included ASA Class, operation time, intraoperative bleeding (intraoperative blood loss was calculated using the difference between the weights of used gauze and the original unused gauze, in addition to the blood volume accumulated in suction bottles), use of TXA, use of drainage tube, and use of autologous blood transfusion from a closed suction drainage system.

\section{Statistical analysis}

Statistical analysis was performed using the $R$ version 3.6 software (America, Lucent Technologies Inc., NJ, USA). Continuous variables were presented in mean \pm standard deviation (SD) or median (min-max), while categorical variables were presented in number and frequency. Descriptive and univariate logistic analyses were initially conducted to compare the demographic characteristics, comorbidities, preoperative laboratory values, and operative variables between the transfusion group and non-transfusion group. A multivariable logistic regression model was used to examine independent risk factors for blood transfusion. A $p$ value of $<0.05$ was considered statistically significant. 


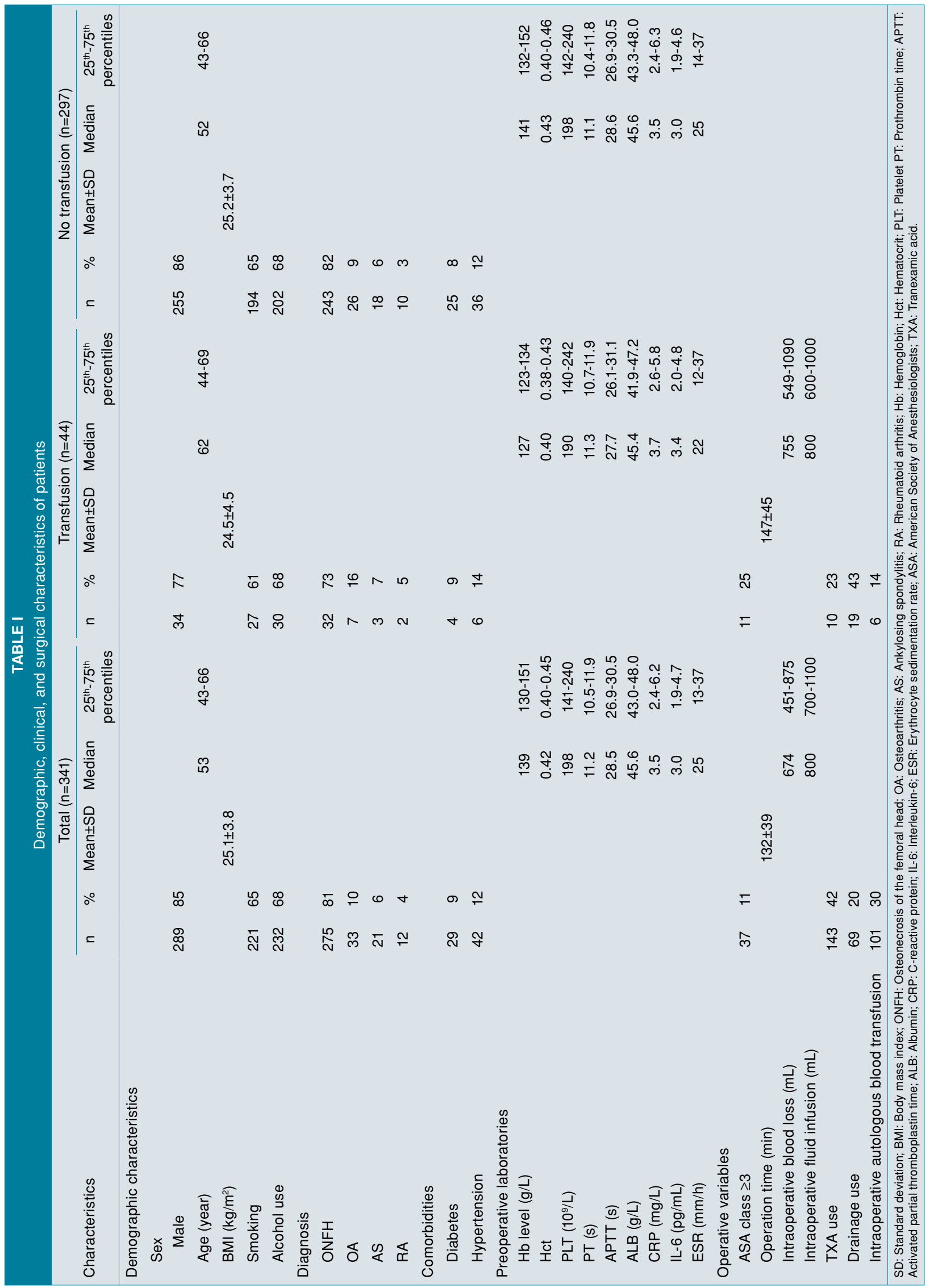




\section{RESULTS}

Of a total of 341 patients, 44 were in the transfusion group, while 297 were in the non-transfusion group with a median age of 62 (range, 44 to 69) years and 73 (range, 64 to 86 ) years, respectively. The incidence of transfusion in SimBTHA was $12.9 \%$. Table I shows the demographic, clinical, and surgical characteristics of the patients.

According to the univariate logistic regression analysis, preoperative hemoglobin $(\mathrm{p}<0.001)$, hematocrit $(\mathrm{p}<0.001)$, albumin $(\mathrm{p}=0.031)$, ASA Class $\geq 3(p=0.002)$, operation time $(p=0.009)$, intraoperative blood loss $(\mathrm{p}<0.001)$, TXA use $(\mathrm{p}=0.007)$, drainage use $(\mathrm{p}<0.001)$, and intraoperative autologous blood transfusion $(\mathrm{p}=0.017)$ were associated with blood transfusion (Table II).

The subsequent multivariate logistic regression analysis showed higher preoperative hemoglobin level (odds ratio [OR]: 0.91, 95\% confidence interval [CI]: 0.86-0.96, $\mathrm{p}=0.001$ ), TXA use (OR: $0.25,95 \% \mathrm{CI}$ : $0.09-0.58, \mathrm{p}=0.002$ ) and intraoperative autologous blood transfusion from a closed suction drainage

\begin{tabular}{|c|c|c|c|}
\hline \multicolumn{4}{|c|}{ TABLE II } \\
\hline Variables & OR & $95 \% \mathrm{Cl}$ & $p$ \\
\hline Age & 1.02 & $1.00-1.04$ & 0.089 \\
\hline \multicolumn{4}{|l|}{ Sex } \\
\hline Male & 0.56 & $0.26-1.22$ & 0.144 \\
\hline Body mass index & 0.95 & $0.88-1.04$ & 0.260 \\
\hline Smoking & 0.84 & $0.44-1.62$ & 0.608 \\
\hline Alcohol use & 1.01 & $0.51-1.99$ & 0.982 \\
\hline \multicolumn{4}{|l|}{ Diagnosis } \\
\hline ONFH & 1.00 (reference) & - & - \\
\hline Osteoarthritis & 2.04 & $0.77-4.88$ & 0.124 \\
\hline Ankylosing spondylitis & 1.27 & $0.29-4.01$ & 0.718 \\
\hline Rheumatoid arthritis & 1.52 & $0.23-6.09$ & 0.600 \\
\hline Diabetes & 1.09 & $0.36-3.29$ & 0.881 \\
\hline Hypertension & 1.14 & $0.45-2.90$ & 0.775 \\
\hline Preoperative level of $\mathrm{Hb}$ & 0.92 & $0.89-0.95$ & $<0.001^{*}$ \\
\hline Preoperative level of Hct & 0.13 & $0.05-0.32$ & $<0.001^{*}$ \\
\hline Preoperative level of PLT & 1.00 & $1.00-1.01$ & 0.855 \\
\hline Preoperative level of PT & 0.93 & $0.78-1.12$ & 0.470 \\
\hline Preoperative level of APTT & 0.95 & $0.87-1.05$ & 0.340 \\
\hline Preoperative level of albumin & 0.92 & $0.85-0.99$ & $0.031^{*}$ \\
\hline Preoperative level of CRP & 1.03 & $0.99-1.07$ & 0.189 \\
\hline Preoperative level of IL-6 & 1.00 & $0.96-1.04$ & 0.972 \\
\hline Preoperative level of ESR & 1.00 & $0.99-1.02$ & 0.807 \\
\hline ASA Class $\geq 3$ & 3.47 & $1.57-7.67$ & $0.002^{*}$ \\
\hline Operation time, $\min$ & 1.01 & $1.00-1.02$ & $0.009^{*}$ \\
\hline Intraoperative blood loss & 1.20 & $1.08-1.32$ & $<0.001^{*}$ \\
\hline Intraoperative fluid infusion & 1.00 & $1.00-1.00$ & 0.687 \\
\hline TXA use & 0.36 & $0.17-0.76$ & $0.007^{*}$ \\
\hline Drainage use & 3.75 & $1.92-7.33$ & $<0.001^{*}$ \\
\hline Intraoperative autologous blood transfusion & 0.34 & $0.14-0.82$ & 0.017 \\
\hline $\begin{array}{l}\text { SimBTHA: Simultaneous bilateral total hip arthro } \\
\text { Osteonecrosis of the Femoral Head; Hb: Hemoglobi } \\
\text { Activated partial thromboplastin time; CRP: C-reactiv } \\
\text { rate; ASA: American Society of Anesthesiologists; TX } \\
\text { regression; * Significant difference. }\end{array}$ & $\begin{array}{l}\text {; OR: Odds rat } \\
\text { Hematocrit; PLT: } \\
\text { ein; IL-6: Interleuk } \\
\text { examic acid; P va }\end{array}$ & $\begin{array}{l}\text { I: Confidence } \\
\text { let; PT: Prothrc } \\
\text { ESR: Erythroc } \\
\text { alculated using }\end{array}$ & $\begin{array}{l}\text { rval; ONFH: } \\
\text { time; APTT: } \\
\text { edimentation } \\
\text { ariate logistic }\end{array}$ \\
\hline
\end{tabular}




\section{TABLE III}

Multivariate logistic regression analysis to identify independent risk factors for transfusion in SimBTHA

$\begin{array}{lccc}\text { Parameter } & \text { OR } & 95 \% \mathrm{Cl} & p \\ \text { Preoperative Hb level } & 0.91 & 0.86-0.96 & 0.001^{*} \\ \text { Preoperative Hct } & 1.06 & 0.18-5.69 & 0.948 \\ \text { Albumin } & 0.96 & 0.89-1.06 & 0.401 \\ \text { ASA Class } & 1.53 & 0.51-4.30 & 0.432 \\ \text { Operation time } & 1.01 & 1.00-1.02 & 0.063 \\ \text { Intraoperative blood loss } & 1.17 & 1.04-1.33 & 0.009^{*} \\ \text { TXA use } & 0.25 & 0.09-0.58 & 0.002^{*} \\ \text { Drainage use } & 3.52 & 1.49-8.32 & 0.004^{*} \\ \text { Intraoperative autologous blood transfusion } & 0.30 & 0.09-0.80 & 0.024^{*}\end{array}$

SimBTHA: Simultaneous bilateral total hip arthroplasty; OR: Odds ratio; Cl: Confidence interval; Hb: Hemoglobin; Hct: Hematocrit; ASA: American Society of Anesthesiologists; TXA: Tranexamic acid; P value calculated using multivariate logistic regression; * Significant difference.

\begin{tabular}{|c|c|c|c|c|c|c|}
\hline Variables & & & & OR & $95 \% \mathrm{Cl}$ & $p$ \\
\hline Preoperative $\mathrm{Hb}$ level & & - & & 0.91 & $0.86-0.96$ & 0.001 \\
\hline Preoperative Hct & & - & $\longrightarrow$ & 1.06 & $0.18-5.69$ & 0.948 \\
\hline Albumin & & + & & 0.96 & $0.89-1.06$ & 0.401 \\
\hline ASA Class & & - & $\rightarrow$ & 1.53 & $0.51-4.30$ & 0.432 \\
\hline Operation time & & $\square$ & & 1.01 & $1.04-1.02$ & 0.063 \\
\hline Intraoperative blood loss & & $-a$ & & 1.17 & $1.04-1.33$ & 0.009 \\
\hline TXA use & $\mapsto$ & & & 0.25 & $0.09-0.58$ & 0.002 \\
\hline Drainage use & & & $\longrightarrow$ & 3.52 & $1.49-8.32$ & 0.004 \\
\hline \multirow[t]{3}{*}{ Intraoperative autologous blood transfusion } & $\longmapsto$ & & & 0.30 & $0.09-0.80$ & 0.024 \\
\hline & 0 & 2 & 3 & & & \\
\hline & & OR & & & & \\
\hline
\end{tabular}

FIGURE 1. The results of multivariate logistic regression analysis.

OR: Odds ratio; Cl: Confidence interval; Hb: Hemoglobin; Hct: Hematocrit; ASA: American Society of Anesthesiologists; TXA: Tranexamic acid.

system (OR: 0.30, 95\% CI: 0.09-0.80, $\mathrm{p}=0.024$ ) were independent protective factors affecting transfusion in patients after SimBTHA. In addition, drainage use (OR: 3.52, 95\% CI: 1.49-8.32, $\mathrm{p}=0.004$ ) and intraoperative blood loss (OR: 1.17, 95\% CI: 1.04-1.33, $\mathrm{p}=0.009$ ) were the independent risk factors for blood transfusion (Table III). The Forest plot is shown in Figure 1.

\section{DISCUSSION}

Blood transfusion after THA is dose-dependent on surgical site infection. ${ }^{[13-15]}$ Transfusion also increases the risk of postoperative venous thromboembolism, transfusions-related acute lung injury, arrhythmias, psychosis, and catheterization. ${ }^{[16-18]}$ Moreover, transfusion appears to increase the rates of in-hospital and one-year postoperative mortality. ${ }^{[11,12,17]}$ Compared to THA, blood transfusion risk of SimBTHA is significantly higher. ${ }^{[19]}$ Only by clarifying the factors of transfusion, we can further optimize and prevent these factors preoperatively and reduce the transfusion rate in SimBTHA. Therefore, it is of utmost importance to elucidate the factors of transfusion in SimBTHA.

In the current study, we included a total of 341 patients who underwent SimBTHA. Univariate and multivariate logistic regression analysis were performed to identify the indicators of both the transfusion group and the non-transfusion group, and 
we concluded that higher preoperative hemoglobin level, TXA use, and intraoperative autologous blood transfusion had protective effects on allogeneic blood transfusion in SimBTHA. In addition, drainage use and intraoperative blood loss were independent risk factors for transfusion.

Higher preoperative hemoglobin level is protective against the need for transfusion in patients after SBTHA, which is consistent with previous studies. ${ }^{[20,21]}$ Salido et al. ${ }^{[22]}$ reported that patients with a preoperative hemoglobin level less than $13 \mathrm{~g} / \mathrm{dL}$ were at a four-fold higher risk of having transfusion compared to those with a hemoglobin level of $>13 \mathrm{~g} / \mathrm{dL}$. Therefore, to reduce the rate and associated risks of blood transfusion, patients with higher hemoglobin can be selected for SimBTHA. For patients with lower preoperative hemoglobin, surgery can be appropriately postponed and treatment to enhance hemoglobin can be performed, until the hemoglobin level is improved.

Furthermore, our study showed that TXA use had protective effects on postoperative blood transfusion in SimBTHA. This is consistent with findings reported in previous studies. ${ }^{[21]}$ Bemelmans et al..$^{[23]}$ reported that low blood transfusion rate was found after implementation of a standardized perioperative TXA protocol for primary hip and knee arthroplasty. In addition, previous studies found that TXA was an effective tool in reducing blood loss and transfusion in simultaneous bilateral total joint arthroplasty. ${ }^{[24,25]}$ However, Partridge et al. ${ }^{[26]}$ reported that, compared to StBTHA, patients undergoing SimBTHA had a greater risk of pulmonary embolism, myocardial infarction, and in-hospital mortality. Therefore, TXA should be used with caution in SimBTHA. The safety of TXA application in SimBTHA needs to be further explored in well-designed studies.

In the present study, autologous blood transfusion from a closed suction drainage system was a protective factor for allogeneic transfusion in SimBTHA. Previous studies have reported varying recommendations in terms of autologous blood transfusion. Palmer et al. ${ }^{[27]}$ suggested that autologous blood transfusion was effective in cases where the indication was infection or fracture and where both femoral and acetabular components were to be revised should be prioritized. A study by Mayer-Rollnik et al. ${ }^{[28]}$ examined the effectiveness of intraoperative autotransfusion as part of a patient blood management program in elective total hip and knee arthroplasty. Recent Association of Anaesthetists guidelines on cell salvage for perioperative blood conservation recommend employing autologous blood transfusion, whenever anticipated blood loss exceeds $500 \mathrm{~mL} .^{[29]}$ We believe that the most important determinants of whether sufficient blood is salvaged for re-infusion is the procedure performed and the indication for surgery. Blood loss is greater in SimBTHA, compared to unilateral THA and, therefore, there may be greater benefits of autologous blood transfusion in SimBTHA. For SimBTHA, blood loss is not the same due to different hospital settings, techniques, and patients. Thus, different centers should decide whether to use autologous blood transfusion as appropriate to maximize the benefit.

Postoperative drainage after THA has always been considered as an effective measure to prevent local hematoma infection, but in recent years, some studies have shown that no drainage tube placement is more beneficial to postoperative recovery of THA. Over blood loss occurs, as the closed negative pressure drainage eliminates the tamponade effect. ${ }^{[30,31]}$ We found that drainage use was independent risk factors for transfusion, which is consistent with previous studies. ${ }^{[21]}$ Therefore, we do not recommend routine use of drainage tubes in SimBTHA. In addition, the amount of intraoperative blood loss was an independent risk factor for transfusion in our study. As intraoperative bleeding is an important component of perioperative blood loss, it may result in receiving a blood transfusion. Thus, reducing the amount of intraoperative blood loss is of utmost importance to reduce the transfusion rate in SimBTHA. Various methods to decrease perioperative blood loss associated with THA have been described in the literature. ${ }^{[2-34]}$

Nevertheless, it is worth mentioning that, as this study consists of young and mostly male patients, findings should be cautiously interpreted and cannot be generalized to the main population of THA. In addition, this study has all limitations inherent to the retrospective design. Also, we only recorded the events of transfusion during hospitalization. Some patients may have received transfusion after discharge to another hospital for short-term inpatient care. Postoperative parameters (e.g., drainage volume, anticoagulants use) may have also affected the transfusion rates; however, these parameters were not considered in our study. Finally, this study is a single-center study with a relatively small sample size, which has a certain impact on the overall representativeness of the statistical results of this study. Further well-designed, multi-center studies are needed to draw more reliable conclusions on this subject. 
In conclusion, the risk factors for transfusion after SimBTHA should be evaluated to establish specific, personalized transfusion risk assessments for each individual patient. For SimBTHA, the main risk factors include intraoperative bleeding and the use of drainage tube, while higher preoperative hemoglobin level, TXA use, and autologous blood transfusion from a closed suction drainage system may reduce the transfusion risk.

\section{Declaration of conflicting interests}

The authors declared no conflicts of interest with respect to the authorship and/or publication of this article.

\section{Funding}

The authors received no financial support for the research and/or authorship of this article.

\section{REFERENCES}

1. Garland A, Rolfson O, Garellick G, Kärrholm J, Hailer NP. Early postoperative mortality after simultaneous or staged bilateral primary total hip arthroplasty: An observational register study from the Swedish Hip Arthroplasty Register. BMC Musculoskelet Disord 2015;16:77.

2. Calabro L, Yong M, Whitehouse SL, Hatton A, de Steiger R, Crawford RW. Mortality and implant survival with simultaneous and staged bilateral total hip arthroplasty: Experience From the Australian Orthopedic Association National Joint Replacement Registry. J Arthroplasty 2020;35:2518-24.

3. Hou JF, Hu C, Zhang Y, Tian LQ, Liu YZ, Zhang C, et al. Cost analysis of staged versus simultaneous bilateral total knee and hip arthroplasty using a propensity score matching. BMJ Open 2021;11:e041147.

4. Guo SJ, Shao HY, Huang Y, Yang DJ, Zheng HL, Zhou YX. Retrospective cohort study comparing complications, readmission, transfusion, and length of stay of patients undergoing simultaneous and staged bilateral total hip arthroplasty. Orthop Surg 2020;12:233-40.

5. Aghayev E, Beck A, Staub LP, Dietrich D, Melloh M, Orljanski W, et al. Simultaneous bilateral hip replacement reveals superior outcome and fewer complications than two-stage procedures: A prospective study including 1819 patients and 5801 follow-ups from a total joint replacement registry. BMC Musculoskelet Disord 2010;11:245.

6. Kamath AF, Austin DC, Derman PB, Clement RC, Garino JP, Lee GC. Saline-coupled bipolar sealing in simultaneous bilateral total knee arthroplasty. Clin Orthop Surg 2014;6:298-304.

7. Bullock DP, Sporer SM, Shirreffs TG Jr. Comparison of simultaneous bilateral with unilateral total knee arthroplasty in terms of perioperative complications. J Bone Joint Surg [Am] 2003;85:1981-6.

8. Bohm ER, Molodianovitsh K, Dragan A, Zhu N, Webster G, Masri B, et al. Outcomes of unilateral and bilateral total knee arthroplasty in 238,373 patients. Acta Orthop 2016;87 Suppl 1:24-30.

9. Edelstein AI, Terzaghi C, Nudelman B, Qin C, Kwasny M, Manning DW. Early response to Warfarin initiation and the risk of venous thromboembolism after total joint arthroplasty. J Am Acad Orthop Surg 2018;26:e90-e97.

10. Jaffer AK, Barsoum WK, Krebs V, Hurbanek JG, Morra $\mathrm{N}$, Brotman DJ. Duration of anesthesia and venous thromboembolism after hip and knee arthroplasty. Mayo Clin Proc 2005;80:732-8.

11. Browne JA, Adib F, Brown TE, Novicoff WM. Transfusion rates are increasing following total hip arthroplasty: Risk factors and outcomes. J Arthroplasty 2013;28(8 Suppl):34-7.

12. Pedersen AB, Mehnert F, Overgaard S, Johnsen SP. Allogeneic blood transfusion and prognosis following total hip replacement: A population-based follow up study. BMC Musculoskelet Disord 2009;10:167.

13. Hart A, Khalil JA, Carli A, Huk O, Zukor D, Antoniou J. Blood transfusion in primary total hip and knee arthroplasty. Incidence, risk factors, and thirty-day complication rates. J Bone Joint Surg [Am] 2014;96:1945-51.

14. Everhart JS, Sojka JH, Mayerson JL, Glassman AH, Scharschmidt TJ. Perioperative allogeneic red blood-cell transfusion associated with surgical site infection after total hip and knee arthroplasty. J Bone Joint Surg [Aml 2018;100:288-94.

15. Taneja A, El-Bakoury A, Khong H, Railton P, Sharma R, Johnston KD, et al. Association between allogeneic blood transfusion and wound infection after total hip or knee arthroplasty: A retrospective case-control study. J Bone Jt Infect 2019;4:99-105.

16. Jiang $\mathrm{T}$, Song $\mathrm{K}$, Yao $\mathrm{Y}$, Pan $\mathrm{P}$, Jiang Q. Perioperative allogenic blood transfusion increases the incidence of postoperative deep vein thrombosis in total knee and hip arthroplasty. J Orthop Surg Res 2019;14:235.

17. Maempel JF, Wickramasinghe NR, Clement ND, Brenkel IJ, Walmsley PJ. The pre-operative levels of haemoglobin in the blood can be used to predict the risk of allogenic blood transfusion after total knee arthroplasty. Bone Joint J 2016;98-B:490-7.

18. Mistry JB, Gwam CU, Naziri Q, Pivec R, Abraham R, Mont $\mathrm{MA}$, et al. Are allogeneic transfusions decreasing in total knee arthroplasty patients? National inpatient sample 20092013. J Arthroplasty 2018;33:1705-12.

19. Slover J, Lavery JA, Schwarzkopf R, Iorio R, Bosco J, Gold HT. Incidence and risk factors for blood transfusion in total joint arthroplasty: Analysis of a statewide database. J Arthroplasty 2017;32:2684-7.

20. Trevisan C, Klumpp R, Auriemma L, Compagnoni R. An algorithm for predicting blood loss and transfusion risk after total hip arthroplasty. Transfus Apher Sci 2018;57:272-6.

21. Cao G, Huang Z, Huang Q, Zhang S, Xu B, Pei F. Incidence and risk factors for blood transfusion in simultaneous bilateral total joint arthroplasty: A multicenter retrospective study. J Arthroplasty 2018;33:2087-91.

22. Salido JA, Marín LA, Gómez LA, Zorrilla P, Martínez C. Preoperative hemoglobin levels and the need for transfusion after prosthetic hip and knee surgery: Analysis of predictive factors. J Bone Joint Surg [Am] 2002;84:216-20.

23. Bemelmans Y, Van Haaren E, Boonen B, Hendrickx $R$, Schotanus M. Low blood transfusion rate after implementation of tranexamic acid for fast- track hip- and knee arthroplasty. An observational study of 5205 patients. Acta Orthop Belg 2021;87:9-16. 
24. Bagsby DT, Samujh CA, Vissing JL, Empson JA, Pomeroy DL, Malkani AL. Tranexamic acid decreases incidence of blood transfusion in simultaneous bilateral total knee arthroplasty. J Arthroplasty 2015;30:2106-9.

25. Wu Y, Yang T, Zeng Y, Si H, Cao F, Shen B. Tranexamic acid reduces blood loss and transfusion requirements in primary simultaneous bilateral total knee arthroplasty: A meta-analysis of randomized controlled trials. Blood Coagul Fibrinolysis 2017;28:501-8.

26. Partridge TCJ, Charity JAF, Sandiford NA, Baker PN, Reed MR, Jameson SS. Simultaneous or staged bilateral total hip arthroplasty? An analysis of complications in 14,460 patients using national data. J Arthroplasty 2020;35:166-71.

27. Palmer AJR, Lloyd TD, Gibbs VN, Shah A, Dhiman $\mathrm{P}$, Booth $\mathrm{R}$, et al. The role of intra-operative cell salvage in patient blood management for revision hip arthroplasty: A prospective cohort study. Anaesthesia 2020;75:479-86.

28. Mayer-Rollnik S, Harms C, Bernasconi L, Hammerer A, Reiss E, Girard T, et al. Evaluation of autologous retransfusion from a closed suction drainage system for patient blood management in elective total hip and knee replacement: A two cohort study. Eur J Anaesthesiol 2020;37:180-6.
29. Klein AA, Bailey CR, Charlton AJ, Evans E, GuckianFisher M, McCrossan R, et al. Association of Anaesthetists guidelines: Cell salvage for peri-operative blood conservation 2018. Anaesthesia 2018;73:1141-50.

30. Niskanen RO, Korkala OL, Haapala J, Kuokkanen HO, Kaukonen JP, Salo SA. Drainage is of no use in primary uncomplicated cemented hip and knee arthroplasty for osteoarthritis: A prospective randomized study. J Arthroplasty 2000;15:567-9.

31. Xu H, Xie J, Lei Y, Huang Q, Huang Z, Pei F. Closed suction drainage following routine primary total joint arthroplasty is associated with a higher transfusion rate and longer postoperative length of stay: A retrospective cohort study. J Orthop Surg Res 2019;14:163.

32. Greenky M, Shaner J, Rasouli MR, Han SB, Parvizi J, Hozack WJ. Intraoperative blood salvage in revision total hip arthroplasty: Who benefits most? J Arthroplasty 2014;29:1298-300.

33. Imai N, Dohmae $\mathrm{Y}$, Suda K, Miyasaka D, Ito T, Endo N. Tranexamic acid for reduction of blood loss during total hip arthroplasty. J Arthroplasty 2012;27:1838-43.

34. Abdelaziz H, Chaabene A, Schulmeyer J, Gehrke T, Haasper C, Hawi N, et al. Intravenous tranexamic acid is associated with safe reduced blood loss and transfusion rate in onestage exchange for infected hip arthroplasty. Jt Dis Relat Surg 2021;32:17-21. 